\title{
INTERPOLATION IN NON-POSITIVELY CURVED KÄHLER MANIFOLDS
}

\author{
CHRISTOPHE MOUROUGANE
}

We extend to any simply connected Kähler manifold with non-positive sectional curvature some conditions for interpolation in $\mathbb{C}$ and in the unit disk given by Berndtsson, Ortega-Cerdà and Seip. The main tool is a comparison theorem for the Hessian in Kähler geometry due to Greene, Wu and Siu, Yau.

\section{INTRODUCTION}

Let $(X, \omega)$ be a Kähler manifold, $(L, h) \rightarrow X$ be a holomorphic hermitian line bundle on $X$, and $\Lambda$ a discrete set of points in $X$. Consider

$$
\mathcal{F}_{\omega, h}:=\left\{f \in H^{0}(X, L) / \int_{X}\|f\|_{h}^{2} d V_{\omega}<+\infty\right\}
$$

and

$$
l_{\Lambda, h}^{2}:=\left\{a \in \Gamma(\Lambda, L) / \sum_{p \in \Lambda}\|a(p)\|_{h}^{2}<+\infty\right\}
$$

We say that the discrete set $\Lambda$ is interpolating for $\mathcal{F}_{\omega, h}$, if for every $a$ in $l_{\Lambda, h}^{2}$ there is a section $f$ in $\mathcal{F}_{\omega, h}$ such that $f_{\mid \Lambda}=a$.

In the complex plane with its usual flat metric, there is a complete characterisation of interpolating sets for pluri-sub-harmonic weights comparable to $|z|^{2}$.

Theorem[Berndtsson and Ortega Cerdà [1], Ortega-Cerdà and Seip [8]]

Consider the trivial line bundle on $\mathbb{C}$ with metric $h_{\Phi}:=||^{2} e^{-\Phi}$ where $\Phi$ is a pluri-sub-harmonic function on $\mathbb{C}$ whose Laplacian is uniformly bounded. A set $\Lambda$ in $\mathbb{C}$ is interpolating for $\mathcal{F}_{h_{\Phi}}$ if and only if it is uniformly separated and

$$
\exists \rho>0, \varepsilon>0 \quad \forall z \in \mathbb{C}, \quad \Delta \Phi(z) \geq \frac{\operatorname{card} B(z, \rho) \cap \Lambda}{\rho^{2}}+\varepsilon .
$$

Here $B(z, \rho)$ is the Euclidean ball of centre $z$ and radius $\rho$.

A simply-connected complete Riemannian manifold with non-positive sectional curvature is called a Cartan-Hadamard manifold. An hermitian complex manifold is called Cartan-Hadamard if its underlying Riemannian space is CartanHadamard.

We prove

Theorem 1. Let $(X, \omega)$ be a Kähler Cartan-Hadamard manifold with sectional curvature bounded from below by $-k^{2}$. Let $\Lambda$ be a discrete set of points in $X$ and $(L, h) \rightarrow X$ be an holomorphic hermitian line bundle on $X$ almost analytic around $\Lambda$ (see definition 团).

If $\Lambda$ is a uniformly $\omega$-separated set such that $\exists \rho>0, \varepsilon>0, \forall z \in X$,

$$
i c_{h}(L)+\operatorname{ricci}(\omega) \geq n \frac{\operatorname{card} B_{\omega}(z, \rho) \cap \Lambda}{\rho^{2}}(1+k \rho \operatorname{coth} k \rho) \omega+\varepsilon \omega
$$


then it is interpolating for $\mathcal{F}_{\omega, h}$.

On the other hand, Lindholm [6] obtained on $\mathbb{C}^{n}$ necessary conditions for interpolation in terms of the Monge-Ampère measure $(i \partial \bar{\partial} \Phi)^{n}$ of the weight. For $n>1$, there is therefore a gap between necessary and sufficient conditions expressed in terms of eigenvalues of the curvature.

Seip also obtained a complete description of interpolating sets in the unit disk with its hyperbolic measure for special weights $-A \log \left(1-|z|^{2}\right)$ in the trivial line bundle in terms of the hyperbolic lower density for $\Lambda$. In the spirit of [9], we define for $(X, \omega)$ a Kähler Cartan-Hadamard manifold with sectional curvature bounded from above by $-1 / \kappa^{2}, \Lambda$ a discrete set of points in $X$, and $x \in X$

$$
D_{\Lambda, \kappa}(x):=\sum_{\substack{p \in \Lambda \\ d_{\omega}(x, p) \geq 1}}-\log \tanh ^{2}\left(\frac{d_{\omega}(x, p)}{2 \kappa}\right) .
$$

We prove

Theorem 2. Let $(X, \omega)$ be a Kähler Cartan-Hadamard manifold with sectional curvature bounded from above by $-1 / \kappa^{2}$ and from below by $-k^{2}$. Assume $\Lambda$ is uniformly $\omega$-separated, and $(L, h)$ is almost analytic around $\Lambda$. Then if

$$
\sup _{X} D_{\lambda, \kappa}<+\infty \text { and } i c_{h}(L)+\operatorname{ricci}(\omega) \geq \varepsilon \omega
$$

for some positive $\varepsilon$, the set $\Lambda$ is interpolating for $\mathcal{F}_{\omega, h}$.

In the next section, we recall some comparison theorem in Riemannian geometry from [4] and [10]. In section 2, we prove that under some assumption relating the complex structure and the metric structure uniformly on $\Lambda$, the uniform separation is necessary. The section 1 is devoted to prove theorem 1 and the section 5 to prove theorem 2 .

Note that using the extension theorem of Ohsawa-Takegoshi-Manivel [7], one can get interpolation results on Stein manifolds, expressed in terms of an equation for the set $\Lambda$.

In the whole work, we will denote by $C$ any constant which only depends on quantities fixed before.

\section{Comparison theorems in Riemannian GeOMetry}

In a Cartan-Hadamard manifold the exponential map is at each point a diffeomorphism. There is no conjugate points. The distance between two points is achieved by a unique geodesic. Hence, the distance function from any point is smooth. Recall that by a result of Wu (see [1] page 100) a Kähler CartanHadamard manifold is Stein. As a consequence of these facts, any holomorphic line bundle on a Kähler Cartan-Hadamard manifold is trivialisable. Nevertheless, it is more convenient to state the results, especially the admissibility condition, in terms of hermitian line bundles instead of pluri-sub-harmonic weights.

By comparison theorem of Rauch (see for example [5] page 76), lower bounds on the sectional curvature yields upper bounds on the Jacobi vector fields and hence on the norm of the differential of the exponential map. By other forms of the comparison theorem (see [2] page 118-119), upper bounds on sectional curvature yields lower bounds on volume. 
We will state the comparison theorem for the Hessian (see [4] Theorem A page 19 ) in a form suitable for our purpose. The proof of it relies on a precise form of the formula for second order variation of arc length, and on a relation between real and complex Hessian on Kähler manifolds (see [11] page 102).

Theorem Let $M$ be a Kähler Cartan-Hadamard manifold of dimension $n$ and constant sectional curvature $k$. Let $f$ be a non-decreasing function on $[0,+\infty[$. Let 0 be a point in $M$. Assume $i \partial \bar{\partial} f\left(d_{M}(0, \cdot)\right)=g\left(d_{M}(0, \cdot)\right) \omega_{M}$ for a function $g$ on $[0,+\infty[$.

Let $X$ a Kähler Cartan-Hadamard manifold of dimension $n$ and sectional curvature bounded from below (resp. from above) by $k$. Let $x_{0} \in X$. Then

$$
i \partial \bar{\partial} f\left(d_{X}\left(x_{0}, \cdot\right)\right) \leq g\left(d_{X}\left(x_{0}, \cdot\right)\right) \omega_{X} .
$$

resp.

$$
i \partial \bar{\partial} f\left(d_{X}\left(x_{0}, \cdot\right)\right) \geq g\left(d_{X}\left(x_{0}, \cdot\right)\right) \omega_{X}
$$

\section{ON UNIFORM SEPARATION}

We generalize the condition on the weight posed in earlier works on interpolation.

Definition 1. Fix $M_{2}$ in $[0,+\infty[$. An hermitian line bundle $(L, h) \rightarrow U$ on an open set $U$ of $\mathbb{C}^{n}$ has an $M_{2}$-regular frame $e$ on $U$ if the weight function $\Phi:=-\log h(e)$ can be written as the sum

$$
\sum_{1 \leq \alpha \leq N}\left|\sigma_{\alpha}\right|^{2}+\Phi_{d e f}
$$

where the $\sigma_{\alpha}$ are holomorphic functions on $U$ with oscillation $\sup _{x, y \in U} \mid \sigma_{\alpha}(x)-$ $\left.\sigma_{\alpha}(y)\right|^{2}$ bounded by $M_{2}$ and all second order real derivatives of the deformation weight $\Phi_{\text {def }}$ are bounded by $-M_{2}$ and $M_{2}$ on $U$.

Definition 2. An hermitian line bundle $(L, h) \rightarrow X$ is said to be regular around $\Lambda$ if there exists positive numbers $r, \lambda, \mu$ and $M_{2}$ such that for all $p \in \Lambda$, there exists a coordinates chart $\Psi_{p}:(U, 0) \subset \mathbb{C}^{n} \rightarrow(V, p) \subset X$ centered at $p$ such that

(i) $\Psi_{p}(U) \supset B_{\omega}(p, r)$

(ii) $\left(\Psi_{p}^{\star} L, h\right)$ has an $M_{2}$-regular frame e on $\Psi_{p}^{-1}\left(B_{\omega}(p, r)\right)$.

(iii) The differential $d \Psi_{p}:\left(T U, \Psi_{p}^{\star} \omega(p)\right) \rightarrow(T V, \omega)$ satisfies $\mu \leq\left\|d \Psi_{p}\right\| \leq \lambda$ on $\Psi_{p}^{-1}\left(B_{\omega}(p, r)\right)$.

Remark first that (iii) implies

$$
\mu d_{e u c l} \leq d_{\omega} \leq \lambda d_{e u c l} \quad \text { on } B_{\omega}(p, r)
$$

where $d_{\text {eucl }}$ is computed with the constant metric $\Psi_{p}^{\star} \omega(p)$ on $U$.

In this section, we will prove the following

Theorem 3. Let $X, L$ and $\Lambda$ as in the introduction. Assume $X$ is a Kähler CartanHadamard manifold and that $(L, h) \rightarrow X$ is regular around $\Lambda$.

Then, if $\Lambda$ is interpolating for $\mathcal{F}_{\omega, h}$, it is uniformly $\omega$-separated.

3.1. On regularity. It can be proved from the fact that $\mathbb{N}$ is interpolating in $\mathbb{C}$ for the weight $|z|^{2}$ that the non-uniformly separated set $\{\sqrt{n}, n \in \mathbb{N}\}$ is interpolating in $\mathbb{C}$ for the weight $\left|z^{2}\right|^{2}$. This explains our restriction on the metric $h$. 
3.2. Mean value estimates. According to [4] (Theorem B page 43), for all $p$ in a Kähler Cartan-Hadamard manifold, for all holomorphic function $f$ on $B_{\omega}(p, r)$ and all $x \in B_{\omega}(p, r / 2)$

$$
|f(x)|^{2} \leq \frac{1}{\operatorname{vol}_{\text {eucl }}\left(B_{\text {eucl }}(r / 2)\right)} \int_{B_{\omega}(x, r / 2)}|f|^{2} d V_{\omega} .
$$

We now assume the hypothesis of theorem 3. The following estimates relies on the simple inequalities for $x, z$ in $B_{\omega}(p, r)$

$$
\begin{aligned}
\left|\sigma_{\alpha}(x)\right|^{2}+2 \Re\left(\overline{\sigma_{\alpha}(x)}\left(\sigma_{\alpha}(z)-\sigma_{\alpha}(x)\right)\right) & =\left|\sigma_{\alpha}(z)\right|^{2}-\left|\sigma_{\alpha}(z)-\sigma_{\alpha}(x)\right|^{2} \\
& \geq\left|\sigma_{\alpha}(z)\right|^{2}-M_{2}
\end{aligned}
$$

and with the coordinates $\left(z_{k}\right)$ provided by $\Psi_{p}$,

$$
\begin{aligned}
\Phi(x)+2 \Re\left(\sum \frac{\partial \Phi}{\partial z_{k}}(x)\left(z_{k}-z_{k}(x)\right)\right) & \geq \Phi(z)-\frac{1}{2} M_{2}(2 n)^{2}|z-x|_{\text {eucl }}^{2} \\
& \geq \Phi(z)-\frac{1}{2} M_{2}(2 n)^{2} \frac{r^{2}}{\mu^{2}} .
\end{aligned}
$$

Then, for all holomorphic section $f$ of $L$ on $B_{\omega}(p, r)$ and all $x$ in $B_{\omega}(p, r / 2)$,

$$
\begin{aligned}
& \|f(x)\|_{h}^{2}=|f(x)|^{2} e^{-\Phi(x)} \\
& \quad \leq C \int_{B_{\omega}\left(x, \frac{r}{2}\right)}\left|f e^{-\sum_{1 \leq \alpha \leq N} \overline{\sigma_{\alpha}(x)}\left(\sigma_{\alpha}(z)-\sigma_{\alpha}(x)\right)-\sum_{1 \leq k \leq n} \frac{\partial \Phi d e f}{\partial z_{k}}(x)\left(z_{k}-z_{k}(x)\right)}\right|^{2} e^{-\Phi(x)} d V_{\omega} \\
& \leq C \int_{B_{\omega}(x, r / 2)}\|f(x)\|_{h}^{2} d V_{\omega} .
\end{aligned}
$$

3.3. Gradient estimates. Let $f$ be a holomorphic section of $L$ on $B_{\omega}(p, r)$ and $x$ in $B_{\omega}(p, r / 4)$. Denote by $s$ any coordinate among the $z_{k}-z_{k}(x)$ centered at $x$. Then

$$
\begin{aligned}
& \frac{\partial}{\partial s}\left(\|f(x)\|_{h}^{2}\right)_{\mid s=0} \\
& =\overline{f(x)} e^{-\Phi(x)}\left(\frac{\partial f}{\partial s}_{\mid s=0}-\frac{\partial \Phi}{\partial s} \mid s=0_{s}\right) \\
& =\overline{f(x)} e^{-\Phi(x)} \frac{\partial}{\partial s}\left(f(s) e^{-\sum_{1 \leq \alpha \leq N} \overline{\sigma_{\alpha}(x)}\left(\sigma_{\alpha}(s)-\sigma_{\alpha}(x)\right)-\frac{\partial \Phi_{d e f}}{\partial s}(0) s}\right)_{\mid s=0} \\
& =\overline{f(x)} e^{-\frac{\Phi(x)}{2}} \int_{|s|=\frac{r}{4 \lambda}} f(s) e^{-\sum_{1 \leq \alpha \leq N} \overline{\sigma_{\alpha}(x)}\left(\sigma_{\alpha}(s)-\sigma_{\alpha}(x)\right)-\frac{\partial \Phi d e f}{\partial s}(0) s} e^{-\frac{\Phi(x)}{2}} \frac{d s}{s}
\end{aligned}
$$

Now, note that thanks to the regularity assumption

$$
\left|f(s) e^{-\sum_{1 \leq \alpha \leq N} \overline{\sigma_{\alpha}(x)}\left(\sigma_{\alpha}(z)-\sigma_{\alpha}(x)\right)-\frac{\partial \Phi_{d e f}}{\partial s}(0) s}\right|^{2} e^{-\Phi(x)} \leq C\|f(s)\|_{h}^{2} .
$$


By the mean value estimate, we get

$$
\left|\frac{\partial}{\partial s}\left(\|f(x)\|_{h}^{2}\right)_{\mid s=0}\right| \leq C \int_{B_{\omega}(p, r)}\|f\|_{h}^{2} d V_{\omega} .
$$

3.4. Uniform separation. Choose an interpolating set $\Lambda$ for $\mathcal{F}_{\omega, h}$. Assume it is not uniformly $\omega$-separated. Then, there exists a subset $\Lambda^{\prime}$ (still interpolating) non-uniformly separated but such that any intersection of three balls among the $B_{\omega}\left(p_{i}, r\right)$ is empty. Hence, by the mean value estimate, the evaluation map

$$
\begin{aligned}
e v_{\Lambda^{\prime}}: \mathcal{F}_{\omega, h} & \rightarrow l_{\Lambda^{\prime}, h}^{2} \\
f & \mapsto f_{\mid \Lambda^{\prime}}
\end{aligned}
$$

is well defined and continuous. By the open mapping theorem, $\Lambda^{\prime}$ is stably interpolating, i.e.

$$
\exists K, \forall a \in l_{\Lambda^{\prime}, h}^{2}, \exists f \in \mathcal{F}_{\omega, h} / f_{\mid \Lambda^{\prime}}=a \text { and } \int_{X}\|f\|_{h}^{2} d V_{\omega} \leq K \sum_{p \in \Lambda^{\prime}}|a(p)|_{h}^{2}<+\infty .
$$

For all $p_{0}$ in $\Lambda^{\prime}$ denote by $f^{p_{0}}$ any stable solution for $a \neq 0$ at $p_{0}$ and $a=0$ on $\Lambda^{\prime}-\left\{p_{0}\right\}$. By the gradient estimate, we infer if $p \in \Lambda^{\prime} \cap B_{\omega}\left(p_{0}, r / 4\right)$,

$$
\left\|f^{p_{0}}\left(p_{0}\right)\right\|_{h}^{2}=\left|\left\|f^{p_{0}}\left(p_{0}\right)\right\|_{h}^{2}-\left\|f^{p_{0}}(p)\right\|_{h}^{2}\right| \leq\left|p-p_{0}\right| \int_{X}\|f\|_{h}^{2} d V_{\omega} \leq \frac{K}{\mu} d_{\omega}\left(p, p_{0}\right)\left\|f^{p_{0}}\left(p_{0}\right)\right\|_{h}^{2}
$$

which contradicts the assumption that $\Lambda^{\prime}$ is not uniformly separated.

\section{INTERPOLATION ON NON-POSITIVELY CURVED MANIFOLDS}

We will prove theorem 1 in this section. We first have to describe the admissible metrics.

Definition 3. An hermitian line bundle $(L, h) \rightarrow U$ on an open set $U$ of $\mathbb{C}^{n}$ has an $M_{2}$-almost analytic frame $e$ on $U$ if the weight function $\Phi:=-\log h(e)$ can be written as the sum

$$
\sum_{1 \leq \alpha \leq N}\left|\sigma_{\alpha}\right|^{2}+\Phi_{d e f}
$$

where the $\sigma_{\alpha}$ are holomorphic functions on $U$ and all second order real derivatives of the deformation weight $\Phi_{\text {def }}$ are bounded by $-M_{2}$ and $M_{2}$ on $U$.

Note that nothing is now assumed on the oscillation of the holomorphic functions $\sigma_{\alpha}$.

Definition 4. An hermitian line bundle $(L, h) \rightarrow X$ is said to be almost analytic around $\Lambda$ if there exists positive numbers $r_{0}$ and $M_{2}$ such that for all $p \in \Lambda$, there exists a coordinates chart $\Psi_{p}:(U, 0) \subset \mathbb{C}^{n} \rightarrow(V, p) \subset X$ centered at $p$ such that

(I) $\Psi_{p}(U) \supset B_{\omega}\left(p, r_{0}\right)$

(II) $(L, h)$ has a $M_{2}$-almost analytic frame e on $\Psi_{p}^{-1}\left(B_{\omega}\left(p, r_{0}\right)\right)$.

(III) The differential $d \Psi_{p}:\left(T U, \Psi_{p}^{\star} \omega(p)\right) \rightarrow(T V, \omega)$ satisfies $\mu \leq\left\|d \Psi_{p}\right\|$ on $\Psi_{p}^{-1}\left(B_{\omega}\left(p, r_{0}\right)\right)$. 
4.1. Local choice. We now make the assumptions of theorem 1. Denote by $2 \delta_{0}:=$ $\inf _{\substack{p, q \in \Lambda \\ p \neq q}}\left(d_{\omega}(p, q), r_{0}\right)$. Let $a \in l_{\Lambda, h}^{2}$ and $p \in \Lambda$. On the ball $B_{\omega}\left(p, \delta_{0}\right)$, consider the holomorphic function

$$
f_{p}:=a(p) \exp \left(\sum_{1 \leq k \leq n} \frac{\partial \Phi_{d e f}}{\partial z_{k}}(p)\left(z_{k}-z_{k}(p)\right)+\sum_{1 \leq \alpha \leq N} \overline{\sigma_{\alpha}(p)}\left(\sigma_{\alpha}-\sigma_{\alpha}(p)\right)\right) .
$$

It is in fact a normal frame at $p$ for $(L, h)$. The following estimates relies on the simple inequalities for $z$ in $B_{\omega}\left(p, \delta_{0}\right)$ which may be regarded as higher dimensional analogues of Riesz formula.

$$
\begin{aligned}
-\left|\sigma_{\alpha}(z)\right|^{2}+2 \Re\left(\overline{\sigma_{\alpha}(p)}\left(\sigma_{\alpha}(z)-\sigma_{\alpha}(p)\right)\right) & =-\left|\sigma_{\alpha}(p)\right|^{2}-\left|\sigma_{\alpha}(z)-\sigma_{\alpha}(p)\right|^{2} \\
& \leq-\left|\sigma_{\alpha}(p)\right|^{2} .
\end{aligned}
$$

and

$$
\begin{aligned}
-\Phi_{\text {def }}(z)+2 \Re\left(\sum_{1 \leq k \leq n} \frac{\partial \Phi_{d e f}}{\partial z_{k}}(p)\left(z_{k}-z_{k}(p)\right)\right) & \leq-\Phi_{\text {def }}(p)+\frac{1}{2} M_{2}(2 n)^{2}|z-p|_{\text {eucl }}^{2} \\
& \leq-\Phi_{\text {def }}(p)+\frac{1}{2} M_{2}(2 n)^{2} \frac{\delta_{0}^{2}}{\mu^{2}}
\end{aligned}
$$

Then, for all $z \in B_{\omega}\left(p, \delta_{0}\right)$,

$$
\left\|f_{p}(z)\right\|_{h}^{2}=\left|f_{p}(z)\right|^{2} e^{-\Phi(z)} \leq C\|a(p)\|_{h}^{2}
$$

where $C$ is a constant independent of $p \in \Lambda$.

4.2. Gluing. Let $\chi$ be a cut-off function defined on $\mathbb{R}^{+}$decreasing, equal to 1 on $[0,1 / 4]$, and to 0 on $[1,+\infty[$. Then,

$$
F:=\sum_{p \in \Lambda} f_{p} \chi\left(\frac{d_{\omega}(p, \cdot)^{2}}{\delta_{0}^{2}}\right)
$$

is a smooth solution of the interpolation problem, holomorphic around $\Lambda$, with the growth condition

$$
\begin{aligned}
\int_{X}\|F\|_{h}^{2} d V_{\omega} & \leq \sum_{p \in \Lambda} \int_{B\left(p, \delta_{0}\right)} C\|a(p)\|_{h}^{2} d V_{\omega} \\
& \leq C \sum_{p \in \Lambda}\|a(p)\|_{h}^{2}<+\infty .
\end{aligned}
$$

Here, we used Rauch theorem to infer that the balls $B\left(p, \delta_{0}\right)$ have volume bounded by a constant independent of $p$.

4.3. Finding holomorphic sections. We will apply the $L^{2}$-estimates existence theorem with a singular weight (see for example [3] theorem 5.1) in order to keep the right values on $\Lambda$. Consider the auxiliary weight

$$
v(z):=n \sum_{q \in \Lambda}\left(1-\frac{d_{\omega}(q, z)^{2}}{\rho^{2}}+\log \frac{d_{\omega}(q, z)^{2}}{\rho^{2}}\right) 1_{B_{\omega}(q, \rho)}(z)
$$


Remark first that,

$$
\int_{X}\|\bar{\partial} F\|_{h}^{2} e^{-v} d V_{\omega}=\int_{X} \sum_{p \in \Lambda}\left|\bar{\partial} \chi\left(\frac{d_{\omega}(p, z)^{2}}{\delta_{0}^{2}}\right)\right|^{2}\left\|f_{p}\right\|_{h}^{2} e^{-v} d V_{\omega}
$$

The term $\left|\bar{\partial} \chi\left(\frac{d_{\omega}(p, z)^{2}}{\delta_{0}^{2}}\right)\right|^{2}$ depends on the differential of the exponential map. Hence it is bounded by a constant independent of the point $p$ by Rauch theorem. Now,

$$
\int_{X}\|\bar{\partial} F\|_{h}^{2} e^{-v} d V_{\omega} \leq C \sum_{p \in \Lambda}\|a(p)\|_{h}^{2} \int_{B_{\omega}\left(p, \delta_{0}\right)-B_{\omega}\left(p, \delta_{0} / 2\right)} e^{-v} d V_{\omega}
$$

We have to estimate $v$ around $p \in \Lambda$. Choose $q$ in $\Lambda$. For $z \in\left(B_{\omega}\left(p, \delta_{0}\right)-\right.$ $\left.B_{\omega}\left(p, \delta_{0} / 2\right)\right) \cap B_{\omega}(q, \rho), d_{\omega}(q, z) \geq d_{\omega}(p, q)-d_{\omega}(z, p) \geq 2 \delta_{0}-\delta_{0} / 2$ if $q \neq p$, and $d_{\omega}(q, z) \geq \delta_{0} / 2$ if $q=p$. Hence,

$$
1-\frac{d_{\omega}(q, z)^{2}}{\rho^{2}}+\log \frac{d_{\omega}(q, z)^{2}}{\rho^{2}} \geq \log \frac{d_{\omega}(q, z)^{2}}{\rho^{2}} \geq \log \frac{\delta_{0}^{2}}{4 \rho^{2}}
$$

Recalling that the number of $q$ in $\Lambda$ such that $z \in B_{\omega}(q, \rho)$ is uniformly bounded because $\Lambda$ is assumed to be uniformly separated and the volume of $B_{\omega}(z, \rho)$ is bounded from above by the volume of a ball radius $\rho$ in the hyperbolic space of dimension $n$ and of sectional curvature $-k^{2}$, one infers that

$$
\int_{X}\|\bar{\partial} F\|_{h}^{2} e^{-v} d x<+\infty
$$

Now, the computation of the curvature is simple because the derivatives of

$$
\left(1-\frac{d_{\omega}(p, z)^{2}}{\rho^{2}}+\log \frac{d_{\omega}(p, z)^{2}}{\rho^{2}}\right)
$$

vanishes along $\partial B_{\omega}(p, a)$.

$$
i \partial \bar{\partial} v=n \sum_{p \in \Lambda}\left(\frac{-i \partial \bar{\partial} d_{\omega}(p, z)^{2}}{\rho^{2}}+i \partial \bar{\partial} \log d_{\omega}(p, z)^{2}\right) 1_{B(p, a)}(z) .
$$

By the comparison theorem with the model $M=\left(\mathbb{C},||^{2}\right)$ and $f=\log$ we get that $\log d_{\omega}(z, p)^{2}$ is a pluri-sub-harmonic function. On the other hand, using the hyperbolic space of curvature $-k^{2}$ as model, with $f=x^{2}$, we get (see [4] page 35)

$$
i \partial \bar{\partial} d_{\omega}(p, z)^{2} \leq\left(1+k d_{\omega}(p, z) \operatorname{coth} k d_{\omega}(p, z)\right) \omega \leq(1+k \rho \operatorname{coth} k \rho) \omega
$$

on $B_{\omega}(p, \rho)$. Hence,

$$
\begin{aligned}
& i c_{h}(L)+i \partial \bar{\partial} v+\operatorname{ricci}(\omega) \\
& \quad \geq i c_{h}(L)+\operatorname{ricci}(\omega)-n \frac{\operatorname{card} B_{\omega}(z, \rho) \cap \Lambda}{\rho^{2}}(1+k \rho \operatorname{coth} k \rho) \omega \\
& \quad \geq \varepsilon \omega .
\end{aligned}
$$

Solving the equation $\bar{\partial} G=\bar{\partial} F$ on $X$ endowed with the complete metric $\omega$ for the line bundle $L$ endowed with the metric $h e^{-v}$, we get a smooth section $G$ of $L$ on $X$ such that $F-G$ is holomorphic and $\int_{X}\|G\|_{h}^{2} e^{-v} d V_{\omega}$ is finite. Now, because 
$v$ is non-positive, $\int_{X}\|G\|_{h}^{2} d V_{\omega}$ is also finite. Furthermore, the estimate of $v$ around $p \in \Lambda$ leads to

$$
\int_{B_{\omega}\left(p, \delta_{0}\right)} \frac{\|G\|_{h}^{2}}{\|z-p\|^{2 n}} \leq C \int_{X}\|G\|_{h}^{2} e^{-v} d x<+\infty,
$$

so that $G$ vanishes on $\Lambda$.

4.4. Comments on the factor $\rho^{2}$. The first remark is an homogeneity property. The quantity

$$
\frac{\operatorname{card} B_{\omega}(z, \rho) \cap \Lambda}{\rho^{2}} \omega
$$

which appears in theorem 11 applied for flat manifolds is equal to the quantity $\frac{\operatorname{card} B_{t \omega}(z, \sqrt{t} \rho) \cap \Lambda}{(\sqrt{t} \rho)^{2}} t \omega$ for all positive $t$.

The second remark is a restriction property, which, together with the criterion for interpolation in $\mathbb{C}$ yields a reason for the factor $\rho^{2}$.

Proposition 1. Consider $\mathbb{C}^{n}$ with its usual flat metric $\omega$ and $(L, h) \rightarrow \mathbb{C}^{n}$ an hermitian line bundle with $M_{2}$-regular frame on a neighbourhood of $\mathbb{C}^{n-1}$ in $\mathbb{C}^{n}$.

If $\Lambda$ is interpolating for $\mathcal{F}_{\omega, h}$ then $\Lambda \cap \mathbb{C}^{n-1}$ is interpolating for $\mathcal{F}_{\omega_{\mid \mathbb{C}^{n-1}}, h_{\mid \mathbb{C}^{n-1}}}$.

Proof Let $a \in l_{\Lambda \cap \mathbb{C}^{n-1}, h}^{2}$. There exists a function $f$ in $\mathcal{F}_{\omega, h}$ achieving $f(p)=a(p)$ for $p \in \Lambda \cap \mathbb{C}^{n-1}$ and $f(p)=0$ for $p \in \Lambda-\mathbb{C}^{n-1}$. To check the growth condition,

$$
\begin{aligned}
& \left|f\left(z^{\prime}, 0\right)\right|^{2} e^{-\Phi\left(z^{\prime}, 0\right)} \\
& \quad \leq \frac{1}{\pi \delta_{0}^{2}} \int_{z_{n} \in D\left(0, \delta_{0}\right)}\left|f\left(z^{\prime}, z_{n}\right) e^{-\sum_{1 \leq \alpha \leq N} \overline{\sigma_{\alpha}\left(z^{\prime}, 0\right)}\left(\sigma_{\alpha}(z)-\sigma_{\alpha}\left(z^{\prime}, 0\right)\right)-\frac{\partial \Phi_{d e f}}{\partial z_{n}}\left(z^{\prime}, 0\right) z_{n}}\right|^{2} e^{-\Phi\left(z^{\prime}, 0\right)} \\
& \quad \leq C e^{1 / 2 M_{2} 2^{2} \delta_{0}^{2}+N M_{2}} \int_{z_{n} \in D\left(0, \delta_{0}\right)}\left|f\left(z^{\prime}, z_{n}\right)\right|^{2} e^{-\Phi\left(z^{\prime}, z_{n}\right)} d x_{n} d y_{n} .
\end{aligned}
$$

We can now apply Fubini theorem because the measure on $\mathbb{C}^{n}$ is a product measure. We get

$$
\int_{\mathbb{C}^{n-1}}\left|f\left(z^{\prime}, 0\right)\right|^{2} e^{-\Phi\left(z^{\prime}, 0\right)} \leq C \int_{\mathbb{C}^{n}}|f(z)|^{2} e^{-\Phi(z)}<+\infty .
$$

\section{INTERPOLATION ON NEGATIVELY CURVED MANIFOLDS}

We will now prove theorem 2 .

The ball $\mathbb{B}(\kappa)$ of radius $\kappa$ in $\mathbb{R}^{2 n}$ endowed with the metric $\frac{4|d z|^{2}}{\left(1-\frac{|z|^{2}}{\kappa^{2}}\right)^{2}}$ has constant sectional curvature $-1 / \kappa^{2}$. The distance between 0 and $z$ is $2 \kappa \operatorname{arctanh} \frac{|z|}{\kappa}$. Hence,

$$
i \partial \bar{\partial} \log \tanh ^{2} \frac{d_{\mathbb{B}(\kappa)}(0, \cdot)}{2 \kappa}=i \partial \bar{\partial} \log |z|^{2} \geq 0 .
$$

Hence, by comparison theorem, for every $p \in X$,

$$
i \partial \bar{\partial} \log \tanh ^{2} \frac{d_{\omega}(p, \cdot)}{2 \kappa} \geq 0 .
$$


Moreover, $v_{p}:=n \log \tanh ^{2} \frac{d_{X}(p, \cdot)}{2 \kappa}$ is negative and has a pole of order $2 n$ at $p$. Consider $v:=\sum_{p \in \Lambda} v_{p}$. By assumption on the density,

$$
v(z) \geq-\sup _{X} D_{\Lambda, \kappa}+\sum_{\substack{p \in \Lambda \\ d_{\omega}(z, p) \leq 1}} v_{p}(z)
$$

Just as we estimated the auxiliary weight in the previous proof, we can estimate $v$ on $B_{\omega}\left(p, \delta_{0}\right)-B_{\omega}\left(p, \delta_{0} / 2\right)$. The rest of the proof follows the same lines as the proof of theorem 1 .

The function $v$ may be compared to a pluri-complex Green function having poles on $\Lambda$.

\section{REFERENCES}

[1] Berndtsson, Bo and Ortega Cerdà, Joaquim, On interpolation and sampling in Hilbert spaces of analytic functions, J. Reine Angew. Math., 464, (1995), 109-128,

[2] Chavel, Isaac, Riemannian geometry - a modern introduction, Cambridge University Press, Cambridge, (1993),

[3] Demailly, Jean-Pierre, Estimations $L^{2}$ pour l'opérateur $\bar{\partial}$ d'un fibré vectoriel holomorphe semi-positif au-dessus d'une variété kählérienne complète, Ann. Sci. École Norm. Sup. (4), 15, (1982), 457-511,

[4] Greene, Robert Everist and Wu, Hung Hsi, Function theory on manifolds which possess a pole, Springer LNM 699, Berlin (1979),

[5] Kobayashi, Shoshichi and Nomizu, Katsumi, Foundations of differential geometry. Vol. II, Interscience Publishers John Wiley \& Sons, Inc., New York-London-Sydney, (1969),

[6] Lindholm, Niklas Ph. D. Thesis, Göteborg

[7] Manivel, Laurent, Un théorème de prolongement $L^{2}$ de sections holomorphes d'un fibré hermitien, Math. Z., 212, (1993), 1, 107-122,

[8] Ortega-Cerdà, Joaquim and Seip, Kristian, Beurling-type density theorems for weighted $L^{p}$ spaces of entire functions, J. Anal. Math., 75, (1998), 247-266,

[9] Seip, Kristian, Beurling type density theorems in the unit disk, Invent. Math., 113, (1993), 21-39,

[10] Siu, Yum Tong and Yau, Shing Tung, Complete Kähler manifolds with nonpositive curvature of faster than quadratic decay, Ann. of Math. (2), 105, (1977), 225-264, and errata in Ann. of Math. (2), 109, (1979), 621-623,

[11] Wu, Hung Hsi, Function theory on noncompact Kähler manifolds, Complex differential geometry, 67-155, Birkhäuser, Basel, (1983), 